\title{
Avaliação sensorial de bebida láctea fermentada com diferentes concentrações de soro de queijo, enriquecida com ferro
}

\author{
Sensorial analysis of fermented dairy beverage with different \\ concentrations of cheese whey, enriched with iron
}

\author{
Vinicius Modesto de Oliveira, ${ }^{*}$ Marco Antonio Sloboda Cortez, ${ }^{* *}$ Mônica Queiroz de Freitas, ${ }^{* *}$ Robson Maia Franco ${ }^{* *}$
}

\begin{abstract}
Resumo
O presente estudo objetivou a avaliação sensorial de três diferentes formulações de bebida láctea fermentada com adição de polpa de frutas, com diferentes concentrações de soro de queijo (10, 30 e 50\%) e enriquecidas com ferro quelato aminoácido. Para isto, foi realizado teste de ordenação de preferência com provadores adultos para determinar a ordem de preferência entre os tratamentos, a partir da diferença mínima significativa pela soma de ordens de Friedman. Também em adultos foi empregado o teste de diferença triangular para detectar adição do ferro. As diferenças entre as amostras foram determinadas utilizando-se o número mínimo de seleções corretas. Como a bebida láctea aromatizada é um alimento muito consumido por crianças, também foi realizado o teste de aceitação por meio de escala hedônica facial, com crianças com idade entre 4 e 6 anos. Todas as avaliações estatísticas foram consideradas no nível de $5 \%$ de probabilidade. As amostras do tratamento com $10 \%$ de soro na formulação foram as menos preferidas pelos julgadores, enquanto que a preferência entre as amostras com 30 e 50\% de soro não diferiam entre si. Já em relação à presença de ferro, os resultados da análise sensorial indicaram que a adição de ferro foi percebida pelos provadores adultos. Já para as crianças, a adição de ferro não foi percebida e o produto teve sua aceitação classificada entre os termos "gostei" e "gostei extremamente". O enriquecimento com ferro para a produção de bebidas lácteas representa uma importante maneira de se veicular um alimento de baixo custo e com características nutricionais importantes.
\end{abstract}

Palavras-chave: bebida láctea, soro de queijo, enriquecimento com ferro, análise sensorial.

\begin{abstract}
The aim of the present study aimed was sensorial evaluation of three different formulations of flavored dairy beverage, with different concentrations of cheese whey $(10,30$ and $50 \%$ ) and enriched with iron. For this, sensory ordination test was accomplished with adult to determine the order preferably among the treatments, using the significant minimum difference of Friedman. Besides, a triangular difference test was used to detect the addition of iron by the adult panelists. The differences among the samples were based on the minimum number of correct selections. Once flavored dairy beverage is a food very consumed by children, a acceptance test was also accomplished using facial scale, with children with age between 4 and 6 years. All statistical evaluations were considered in the level of $5 \%$ of probability. Samples of the treatment with $10 \%$ of whey in the formulation were the least favorite ones, while the preference between samples with 30 and $50 \%$ of whey didn't differ to each other. In relation to iron presence, the sensorial results indicated that the addition of iron was noticed by adult panelists. To the children, the ion addition was not noticed and the product had your acceptance classified between the terms "I liked" and "I liked extremely". The enrichment with iron in the production of dairy beverages represents an important way to commercialize a low cost food, with important nutritional characteristics.
\end{abstract}

Keywords: dairy beverage, cheese whey, enrichment with iron, sensorial analyses.

\section{Introdução}

O soro de queijo é o líquido residual obtido a partir da coagulação do leite destinado à fabricação de queijos ou de caseína (Brasil, 2005a). É considerado o principal subproduto da indústria de laticínios e em sua composição existe boa quantidade de excelentes nutrientes como a lactose $(5,0 \%)$ e proteínas de elevado valor biológico $(0,9 \%)$, tornando-se uma excelente fonte de proteínas a um baixo custo (Mawson, 1994). No Brasil, tal subproduto vem sendo produzido em quantidades cada vez maiores devido ao incremento do consumo de queijos.

Em relação às anemias nutricionais, a anemia ferropriva representa $50 \%$ das ocorrências em todo o mundo, atingindo

* Programa de Pós-Graduação em Medicina Veterinária (Mestrado) Higiene Veterinária e Processamento Tecnológico de Produtos de Origem Animal - Faculdade de Veterinária - Universidade Federal Fluminense. Rua Vital Brazil Filho, 64, CEP 24230-340.

** Departamento de Tecnologia dos Alimentos - Faculdade de Veterinária - Universidade Federal Fluminense. Rua Vital Brazil Filho, 64, CEP 24230-340.

Autor para correspondência: Marco Antonio Sloboda Cortez. E-mail: macortez@vm.uff.br. 
as mais diversas faixas etárias (Batista Filho e Ferreira, 1996). Segundo Silva (2000), a busca de alternativas para o combate à carência de ferro, que melhor se adaptem às condições de cada região, tem sido motivo de estudo. Torres et al. (1996) concluíram pela viabilidade e eficácia da fortificação do leite fluido como medida de intervenção no combate à carência de ferro em pré-escolares.

Devido a grande oferta de soro de queijo no Brasil, suas características nutricionais, potencial poluidor e baixo custo, o soro torna-se uma importante matéria-prima que pode ser utilizada na produção de bebida láctea. Esta por sua vez pode ser utilizada como veículo de ferro, quando enriquecida por esse oligoelemento, no combate à anemia ferropriva (Silva, 2000).

Sendo assim, o objetivo do presente trabalho foi a avaliação sensorial de bebida láctea fermentada, aromatizada com póla de morango, produzida com três diferentes concentrações de soro de queijo (10, 30 e $50 \%$ ) e enriquecida com ferro quelato aminoácido.

\section{Material e métodos}

O produto desenvolvido teve como ingredientes: soro de queijo, leite integral, sacarose, polpa de morango, ferro aminoácido quelato e cultura láctica contendo Streptococcus salivarius ssp. thermophilus e Lactobacillus delbrueckii ssp. bulgaricus. O leite utilizado foi proveniente de criadores da bacia leiteira dos municípios de Cachoeiras de Macacu, Magé e adjacências no estado do Rio de Janeiro, sendo classificado como tipo B. A bebida láctea foi produzida em um laticínio da região, após a obtenção do soro de queijo-minas frescal produzido no próprio estabelecimento e a realização de análises de rotina do leite e do soro para garantir a qualidade do produto.

Na elaboração da bebida láctea utilizou-se a técnica descrita por Ferreira (1996). Foram utilizadas três formulações diferentes para a elaboração da bebida láctea fermentada, sendo que os ingredientes que variaram nas concentrações adicionadas foram o soro e o leite, além da adição ou não de ferro. Desta forma, foram determinadas três bases lácteas diferentes, que foram codificadas como A10, A30 e A50 para as amostras contendo 10, 30 e $50 \%$ de soro respectivamente. A seguinte formulação foi utilizada: polpa (10L), aroma $(0,38 \mathrm{~L})$, corante $(0,32 \mathrm{~L})$, açúcar $(110 \mathrm{~g})$, estabilizante $(3,0 \mathrm{~g})$ e sorbato $(0,46 \mathrm{~g})$.

A adição de ferro ao produto foi padronizada para as três amostras (10, 30 e $50 \%$ de soro de queijo) seguindo os parâmetros propostos por Brasil (1998) que considera como alimento enriquecido ou fortificado aquele que fornecesse no mínimo 15\% da IDR (Ingestão Diária de Alimentos) em 100mL do produto pronto para o consumo. No presente estudo foi utilizado como parâmetro para fortificação a IDR de crianças entre quatro a seis anos de idade, que é de 6mg (Brasil, 2005b).

A bebida láctea foi avaliada em triplicata quanto aos seguintes aspectos físico-químicos: $\mathrm{pH}$, umidade, proteínas e lipídios de acordo com Métodos Analíticos Oficiais Físico-Químicos para Controle de Leite e Produtos Lácteos (Brasil, 2003). As análises foram realizadas no sétimo dia de estocagem.
As análises sensoriais foram realizadas com provadores adultos, universitários da Faculdade de Veterinária da Universidade Federal Fluminense e com crianças, estudantes da rede pública de ensino municipal do Rio de Janeiro, residentes no bairro do Andaraí e adjacências. Os testes foram conduzidos objetivando estimar a preferência dos adultos quanto à concentração de soro adicionada às bebidas lácteas (10, 30 e 50\% de soro) e verificar se a adição de ferro produziria modificação sensorial perceptível pelos mesmos, utilizandose os testes de Ordenação da Preferência e Diferença pelo Método triangular respectivamente. Além de estimar a aceitação da bebida láctea entre crianças. Foram calculadas as diferenças mínimas significativas utilizando-se níveis de probabilidade de $5,0 \%$.

Nos testes realizados com crianças empregou-se uma categoria de teste de aceitação conhecido como Teste Hedônico, caracterizado pelo conhecimento do status afetivo dos produtos, utilizando-se uma escala hedônica facial de cinco pontos. O teste foi dividido em duas sessões, uma para o tratamento com $50 \%$ de soro adicionado de ferro (A50F) e outra para o tratamento com $50 \%$ sem a adição do ferro (A50), utilizando-se provadores na faixa etária de 4 a 7 anos de idade.

\section{Resultados e discussão}

Os resultados das análises físico-químicas realizadas no sétimo dia de estocagem das bebidas lácteas elaboradas com diferentes níveis de soro estão descritas na Tabela 1.

Tabela 1: Resultados médios de gordura, proteína, umidade e $\mathrm{pH}$ das bebidas lácteas elaboradas com diferentes concentrações de soro

\begin{tabular}{ccccc}
\hline & $\begin{array}{c}\text { Gordura } \\
(\% \mathrm{~m} / \mathrm{m})\end{array}$ & $\begin{array}{c}\text { Proteína } \\
(\% \mathrm{~m} / \mathrm{m})\end{array}$ & $\begin{array}{c}\text { Umidade } \\
(\% \mathrm{~m} / \mathrm{m})\end{array}$ & $\mathrm{pH}$ \\
\hline A10 & 2,6 & 2,08 & 83,16 & 4,13 \\
A30 & 2,0 & 2,02 & 83,51 & 4,13 \\
A50 & 1,6 & 1,65 & 85,06 & 4,20 \\
\hline
\end{tabular}

De acordo com a legislação brasileira pertinente (Brasil, 2005a), bebidas lácteas fermentadas devem apresentar no mínimo $2 \mathrm{~g} / 100 \mathrm{~mL}$ de matéria gorda de origem láctica, o que ocorreu com as amostras A10 e A30, estando, desta forma, dentro dos padrões recomendados. Entretanto, a amostra A50, que apresentou $1,6 \%$, não se enquadrou neste padrão de identidade e qualidade, não podendo, portanto, receber tal denominação ou ser comercializada como tal. Tal fato se deu principalmente devido à maior adição de soro, diluindo mais o teor de gordura, o que pode ser comprovado pela determinação do teor de umidade das amostras dos diferentes tratamentos.

A diferença entre os valores de umidade foi mais evidente entre as formulações $A 10$ e A50, visto que o percentual de soro de queijo adicionado em $A 50$ foi muito superior às amostras $\mathrm{A} 10$ e A30, que obtiveram valores de umidade praticamente semelhantes. Isto pode ser atribuído ao fato de que a substituição do leite pelo soro, em maiores quantidades, aumenta o teor relativo de água no produto à medida que há diminuição dos constituintes sólidos presentes 
no leite. Segundo os padrões fixados para bebida láctea fermentada (Brasil, 2005a), não existem valores determinados para umidade neste alimento, apesar disso, os valores encontrados para as três formulações estão próximas ao encontrado por Silva (2000), que foi de $82 \%$ para bebida láctea fermentada com adição de polpa de manga e produzida com aproximadamente $30 \%$ de soro.

Constatou-se também que à medida que a quantidade de soro adicionado foi aumentada, houve diminuição no teor de proteínas. O teor mínimo de proteína para bebida láctea com leite fermentado é de 1,6 g/ 100mL, de acordo com Brasil (2005a). Desta forma, em relação aos índices protéicos obtidos, as três amostras produzidas se enquadram no Regulamento Técnico de Identidade e Qualidade do produto, podendo receber a denominação de Bebida Láctea com logurte e polpa de frutas.

Os resultados da determinação de $\mathrm{pH}$ durante o período de estocagem a temperatura de $4^{\circ} \mathrm{C}$ mostraram uma pequena variação ao longo dos 35 dias, com um valor médio para os três tratamentos em torno de 4,19. Esta variação não descaracteriza o padrão do produto, uma vez que o $\mathrm{pH}$ normalmente encontrado para bebidas lácteas está em torno de 4,5 .

$\mathrm{Na}$ análise sensorial das bebidas lácteas, a diferença de soma de ordens entre as amostras A10, A30 e A50 quanto à preferência dos julgadores pode ser observada na Tabela 2. Segundo o teste de soma de ordens de Friedman, de acordo com o número de amostras diferentes e o número de provadores neste experimento, o valor de Diferença Mínima Significativa (DMS) para se obter diferença significativa entre tratamentos ao nível de $5 \%$ foi de 21 .

Tabela 2: Diferença de soma de ordens entre as amostras $A 10, A 30$ e $A 50$ quanto à preferência dos julgadores obtida pelo Teste de Ordenação da Preferência

\begin{tabular}{cc}
\hline Diferença da soma de ordens (DMS) & Módulos da diferença \\
\hline A10 - A30 & $29,0(\mathrm{~s})$ \\
A10 - A50 & $37,0(\mathrm{~s})$ \\
A30 - A50 & $8,0(\mathrm{~ns})$ \\
\hline
\end{tabular}

(s) = significativo $(\mathrm{ns})=$ não significativo. DMS - Diferença Mínima Significativa $(p<0,05)=21$

Observou-se que as amostras formuladas com 30 e $50 \%$ de soro (A30 e A50) não diferiram significativamente entre si $(p<0,05)$, sendo consideradas como as preferidas pelos julgadores, enquanto a amostra com $10 \%$ de soro (A10) diferiu significativamente das demais, sendo considerada como a menos preferida pelos julgadores. Tal resultado pode ser atribuído ao fato da amostra A10 parecer menos doce do que A30 e A50, pois grande parte dos provadores indicou tal diferença no espaço reservado aos comentários subjetivos da ficha de avaliação. Logo, constatou-se que o aumento na adição de soro é diretamente proporcional ao atributo doçura, o que pode ser explicado pela grande quantidade de lactose encontrada no soro de queijo (Mawson, 1994), responsável pela preferência dos provadores.
No teste de diferença pelo método triangular foram servidas as amostras contendo $50 \%$ de soro de queijo enriquecidas com ferro quelato aminoácido (A50F) e não enriquecida com ferro quelato aminoácido (A50). Realizaram-se 90 degustações, sendo obtidos 26 julgamentos errados e 64 certos. De acordo com Roessler et al. (1978), o Número Mínimo de Seleções Corretas (NMSC) para estabelecer diferença significativa ao nível de $5 \%$ seria de 38 acertos. Desta forma, a presença de ferro quelato aminoácido na concentração de $1,5 \mathrm{mg}$ ferro/ $100 \mathrm{~mL}$ do produto acabado, que corresponde a $25 \%$ da IDR de crianças entre quatro e seis anos de idade, foi percebida por adultos.

Para a escala hedônica facial foram atribuídos conceitos às figuras que variam da esquerda para a direita em "desgostei extremamente", "desgostei", "indiferente", "gostei", "gostei extremamente". Além disso, foram atribuídos valores numéricos que variam de -2 para "desgostei extremamente" a +2 para "gostei extremamente", sendo o "indiferente" igual a zero. Desta forma, a média do escore de aceitação obtida para a amostra A50F foi de 1,9, enquanto que para o teste com a amostra A50 foi de 1,8, correspondendo a $97,0 \%$ e $91,5 \%$ respectivamente. O que permitiu concluir que ambos os resultados foram positivos para provadores com idade entre 4 e 7 anos, cuja aceitação dos produtos variou entre os termos hedônicos "gostei" e "gostei extremamente".

Conforme pôde ser verificado pelos resultados, a presença ou não do ferro, não exerceu influência negativa na aceitabilidade da bebida láctea pelas crianças, além disso, a aceitação dos produtos foi elevada, indicada pela atitude receptiva das crianças, com freqüentes solicitações de repetição.

\section{Conclusão}

Comprovou-se que há diferença significativa entre os padrões físico-químicos das amostras produzidas, apenas modificando seus percentuais de soro de queijo.

As formulações contendo 10 e $30 \%$ de soro foram consideradas aptas para a produção pela indústria, uma vez que sua composição está dentro dos padrões designados pela legislação. A amostra com $30 \%$ de soro foi considerada a ideal para a produção, pois além de se enquadrar nos parâmetros físico-químicos estabelecidos, apresentou percentual de proteína elevado, próximo ao da amostra com $10 \%$ de soro e não diferiu significativamente $(p<0,05)$ da amostra com $50 \%$ de soro quanto à preferência dos julgadores, que consideraram as amostras com 30 e $50 \%$ as mais preferidas. Em relação à adição de ferro, apenas os provadores adultos foram capazes de perceber a presença deste elemento. A bebida láctea com ferro foi prontamente aceita pelos provadores de idade entre 4 e 6 anos, que a classificaram com os termos "gostei" e "gostei extremamente".

Desta forma, concluiu-se que a utilização do soro de queijo na elaboração de bebidas lácteas fermentadas é uma das formas de aproveitar as características nutricionais deste subproduto. Além disso, a bebida láctea mostrou-se um excelente veículo de ferro quando este for adicionado ao produto na concentração adequada. 


\section{Referências}

BATISTA FILHO, M.B.; FERREIRA, L.O.C. Prevenção e tratamento da anemia nutricional ferropriva: novos enfoques e perspectivas. Caderno de Saúde Pública, v. 12, p. 411-415, 1996.

BRASIL. Agência Nacional de Vigilância Sanitária. Portaria no 31, de 13 de janeiro de 1998. Aprova o Regulamento Técnico referente a Alimentos Adicionados de Nutrientes Essenciais. Brasília, DF, 1998.

BRASIL. Agência Nacional de Vigilância Sanitária. RDC no 269, de 22 de setembro de 2005. Aprova o Regulamento Técnico sobre a Ingestão Diária Recomendada (IDR) de Proteína, Vitaminas e Minerais. Brasília, DF, 2005b.

BRASIL. Ministério da Agricultura e do Abastecimento, Secretaria de Defesa Agropecuária. Departamento de Inspeção de Produtos de Origem Animal. Regulamento Técnico de Identidade e Qualidade de Bebidas Lácteas. Brasília, DF, 2005a.

BRASIL. Ministério da Agricultura. Laboratório Nacional de Referência Animal. Métodos Analíticos Oficiais Físico-Químicos para Controle de Leite e Produtos Lácteos. Instrução Normativa sda no 22 , de 14 de abril de 2003.
FERREIRA, C.L.L.F. Produtos lácteos fermentados (aspectos bioquímicos e tecnológicos). Viçosa: Universidade Federal de Viçosa, 1996. $96 \mathrm{p}$.

MAWSON, A.J. Bioconversations for whey utilization and waste abatement. Bioresource Technology, v. 47, p. 195-203, 1994.

ROESSLER, E.B.; PANGBORN, R.M. SIDEL, J.L.; STONE, H. Expanded statistical tables for estimating significance in paired-preference, paired-difference, duo/trio and triangle tests. Journal of Food Science, v. 43, n. 3, p. 940-943, 1978.

SILVA, M.R. Efeito de uma bebida Láctea fermentada e fortificada com ferro no estado nutricional de ferro em pré-escolares. Dissertação (Mestrado em Ciência e Tecnologia de Alimentos Viçosa: UFV, 2000.75p.) - Universidade Federal de Viçosa, 2000.

TORRES, M.A.A.; LOBO, N.F.; SATO, K.; SOUZA QUEIROZ, S. Fortificação do leite fluido na prevenção e tratamento da anemia carencial ferropriva em crianças menores de 4 anos. Revista de Saúde Pública, v. 30, p. 350-357, 1996. 